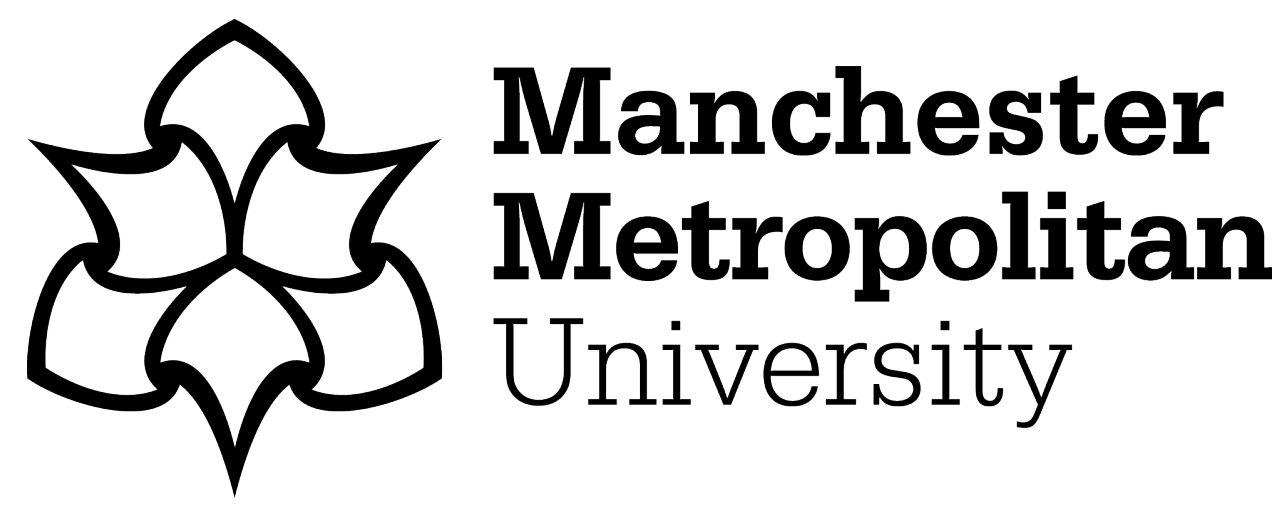

Chesterton, Lorna, Stephens, Melanie, Clark, Andrew and Ahmed, Anya ORCID logoORCID: https://orcid.org/0000-0002-6164-0656 (2021) A systematic literature review of the patient hotel model. Disability and Rehabilitation, 43 (3). pp. 317-323. ISSN 0963-8288

Downloaded from: https://e-space.mmu.ac.uk/625886/

Version: Accepted Version

Publisher: Taylor \& Francis

DOI: https://doi.org/10.1080/09638288.2019.1628314

Please cite the published version 


\title{
A systematic literature review of the patient hotel model
}

\author{
Lorna Chesterton (D), Melanie Stephens, Andrew Clark and Anya Ahmed \\ School of Health and Society, University of Salford, Salford, UK
}

\begin{abstract}
Background: The patient hotel model was developed in Northern Europe as a response to increased demand for health and wellbeing services. According to current literature the patient hotel model is a concept of care provision which combines non-acute hospital care with hospitality to afford patients/ guests increased satisfaction and security whilst benefitting from evidenced based care.

Objective(s): This paper evaluates the concept of the patient hotel model. It presents the findings of a systematic review of existing literature evaluating the benefits such a model can bring to healthcare services and reports on the efficacy in terms of cost to health service providers, and health outcomes to patients/guests. The authors' aimed to complete a meta-analysis of the data, but were unable to, due to the diversity in the descriptions, service provisions, and client group.
\end{abstract}

Design: The Preferred Reporting Items for Systematic Reviews and Meta-Analyses (PRISMA) statement was used to conduct and report this systematic review. In order to focus the research question, the PICO (Population; Intervention; Comparison and Outcome) framework was used to develop a strategy in literature searching, to ensure systematic rigor.

Data sources/review methods: Cochrane Database of Systematic Reviews, OVID MEDLINE, CINAHL, Embase, ScienceDirect, Web of Science, and Scopus databases were used to search for randomized controlled trials, quasi experimental studies, quantitative and qualitative studies conducted between January 1st 2008 and August 9th, 2018, published in a peer reviewed journal in English or which provided an abstract in English. Citation searches and hand searches were also conducted.

Results: 8,693 papers were retrieved and from abstract screening 68 full-text articles were assessed for eligibility by applying an inclusion and exclusion criteria. Seven articles were retained for quality assessment. Methodological rigor was appraised using accepted criteria for the evaluation of research. On appraisal, one systematic review, one Randomized Clinical Trial, two qualitative studies, one quantitative survey, one retrospective analysis of services, and one comparative analysis paper were included for data synthesis. The functionality of the patient hotel model differed across the six countries that reported on them in the included studies, from oncology care, medical care, post-acute rehabilitation and perioperative care. The studies included in this review broadly focused on the themes of patient experience and/or cost, with the intention of informing future service provision. Studies relating to cost efficacy looked at the potential financial savings which could be realized through adopting the patient hotel model. The appraised studies found positive benefits of adopting the patient hotel model, both in terms of cost and patient satisfaction. One study explored the role of nurses in a patient hotel.

Conclusions: The lack of consistent definition, diversity in the descriptions, service provisions, and client groups meant that the results could only be systematically reviewed and not synthesized into a metaanalysis. The inconsistencies in labeling and description also have implications for the review process, as studies adopting more abstract classification of the patient hotel model may not have been included in the review. On balance, the appraised evidence appears to suggest that there are positive benefits to patients, nurses and healthcare providers. However further research of greater rigor is needed to provide a better understanding of these outcome measures.

- IMPLICATIONS FOR REHABILITATION

- Patient hotels have particular relevance to the field of rehabilitation as they have a strong ethos of promoting self-care and independence, facilitated through greater freedom and family involvement.

- The patient hotel model has the potential to impact healthcare provision on the global stage, but there is no universal definition, making evidence evaluation difficult.

- The patient hotel model combines the concepts of health and hospitality and could potentially provide a cost-effective alternative to healthcare, with positive outcomes for patient experience as well as patient health.

- There is a need to explore new systems of care delivery which provide increased patient satisfaction, and a seamless continuum of care at the acute and primary care interface.

- This paper examines the patient hotel model of care, and its merits in terms of care provision, patient satisfaction and service efficiency, contributing to the embryonic literature in this field.

\section{ARTICLE HISTORY}

Received 8 November 2018

Revised 3 June 2019

Accepted 3 June 2019

\section{KEYWORDS}

Patient hotel; recovery hotel; healthcare hotel; hospital hotel 


\section{What is already known on this subject?}

- Patient hotels are an established resource in many Scandinavian countries, yet little research underpins their efficacy.

- Patient flow through hospitals is known to be interrupted by delayed transfers of care, and some health service providers are looking at the patient hotel model as a cost-effective way of dealing with this problem.

- Patient satisfaction is becoming a recognized driver in the commissioning of health services, and there is a need to explore new systems of care delivery which delivers this requirement.

\section{What this paper adds}

- This paper reviews the evidence base for the emergent patient hotel model and suggests areas for future research.

- The patient hotel model combines the concepts of health and hospitality and could potentially provide a cost-effective alternative to healthcare, with potentially positive outcomes for patient experience as well as patient health.

\section{Introduction}

There is global interest in transforming the landscape of care delivery in order to develop a more efficient transition at the acute and primary care interface. The patient hotel model may provide an alternative approach to lengthy periods of hospitalization and could potentially expedite discharge through intensive rehabilitation in a comfortable environment. Indeed, this model has been trialed widely, in various guises, across Europe and North America, although the literature on the subject remains limited. The concept of the patient hotel combines an evidencebased approach to healthcare in environments that appear to mimic or reproduce those of tourist-style hotels, in part because findings suggest that environmental stimuli has a positive influence on the health and well-being of patients and directly affects their security needs [1]. Certainly, patient experience is now seen as a measure of quality in healthcare provision and is recognized as a major driver in the commissioning of services [2-4]. However, commissioning decisions must be balanced against key deliverables in terms of health outcomes, and cost effectiveness, presenting a need to evaluate alternative approaches.

\section{The patient hotel model}

Defining a patient hotel appears problematic in the literature, with no commonality in terms of description, client group or treatment provision [5] The most commonly cited definition comes from the systematic review by the National Knowledge Center for Health Services [6, p.9]:

A temporary, voluntary accommodation where the patient has greater freedom to visit with relatives than in a regular hospital ward. The use of patient hotels requires a connection to a stay in hospital... The regulation of patient hotels still permits some treatment.

However, the definition offered by Holte et al. [6] does not acknowledge the environmental aspects of the patient hotel model, which is arguably a fundamental element when differentiating between traditional hospital accommodation and patient hotel accommodation. Indeed, the aesthetical environment and increased privacy are known to reduce stress levels and increase satisfaction [7]. Pizam [8] asserts that there are positive impacts upon recovery in environments where hospitality is combined with healthcare. Certainly, the need to experience a sense of security seems to be an important requirement, particularly in patients with serious illnesses such as cancer [3]. Moreover, given that patient satisfaction is now an indicator of clinical quality [9] the esthetics of accommodation require greater discussion by stakeholders. It is also important to note that the creation of a hospitality styled environment also benefits staff satisfaction, by stimulating employee pride [10].

A patient hotel is described by Docrates as a building which operates as accommodation and includes healthcare provision and should be connected to a hospital stay or episode of care [11]. In some cases, a patient hotel operates as on-site accommodation for pre and post-operative patients, who live a distance from the hospital and for whom the burden of travel may compromise health [12]. In this situation guests generally receive care from a relative or friend and can access emergency treatment if a health crisis occurs, but no direct care is offered by the hotel establishment and the resources of the hospital are not impacted [6]. A central tenet to the patient hotel model is the emphasis placed on the importance of independence, which is facilitated through greater freedom and family involvement, and an emphasis on a self-care framework and greater patient autonomy [6,13]. Additionally, patient hotels are also designed to cater for specific patient groups, such as maternity provision [14] or perioperative care [5].

In order to conceptualize the patient hotel model, the authors propose the following working definition, which was used to guide this systematic review:

Short term accommodation offering an aesthetic and comfortable environment in which patients may receive restorative care following or preceding hospital treatment. Patient hotels offer non-acute care to hospital patients, encouraging a philosophy of independence and allows relatives and friends to be actively involved in care.

The remainder of this paper presents the findings of the systematic review which has been undertaken and evaluates the benefits that the patient hotel model can bring to healthcare provision and report on the efficacy in terms of cost and health outcomes to patients/guests.

\section{Methods}

\section{Design}

This Systematic review was carried out in accordance with the Preferred Reporting Items for Systematic Reviews and MetaAnalyses (PRISMA) statement, an evidence-based minimum set of items for reporting in systematic reviews and meta-analyses [15]. PRISMA attends to the reporting of reviews, appraising randomized trials, but may also be used as a foundation for recording systematic reviews of further kinds of research, mainly evaluations of interventions [15]. The reporting of this systematic review can be viewed in Figure 1 which documents the search process followed.

The PICO (Population; Intervention; Comparison and Outcome) framework was used to develop a strategy in literature searching, to ensure systematic rigor [16].

\section{Eligibility criteria}

Before the literature search was conducted, inclusion and exclusion criteria were applied to the research question [17]. The review excluded studies from low income countries as results needed to be comparable across healthcare systems. Studies specializing in end of life care, outpatient clinics, pediatric or child healthcare, and those acting as temporary accommodation for homeless individuals were also excluded. This was because the 


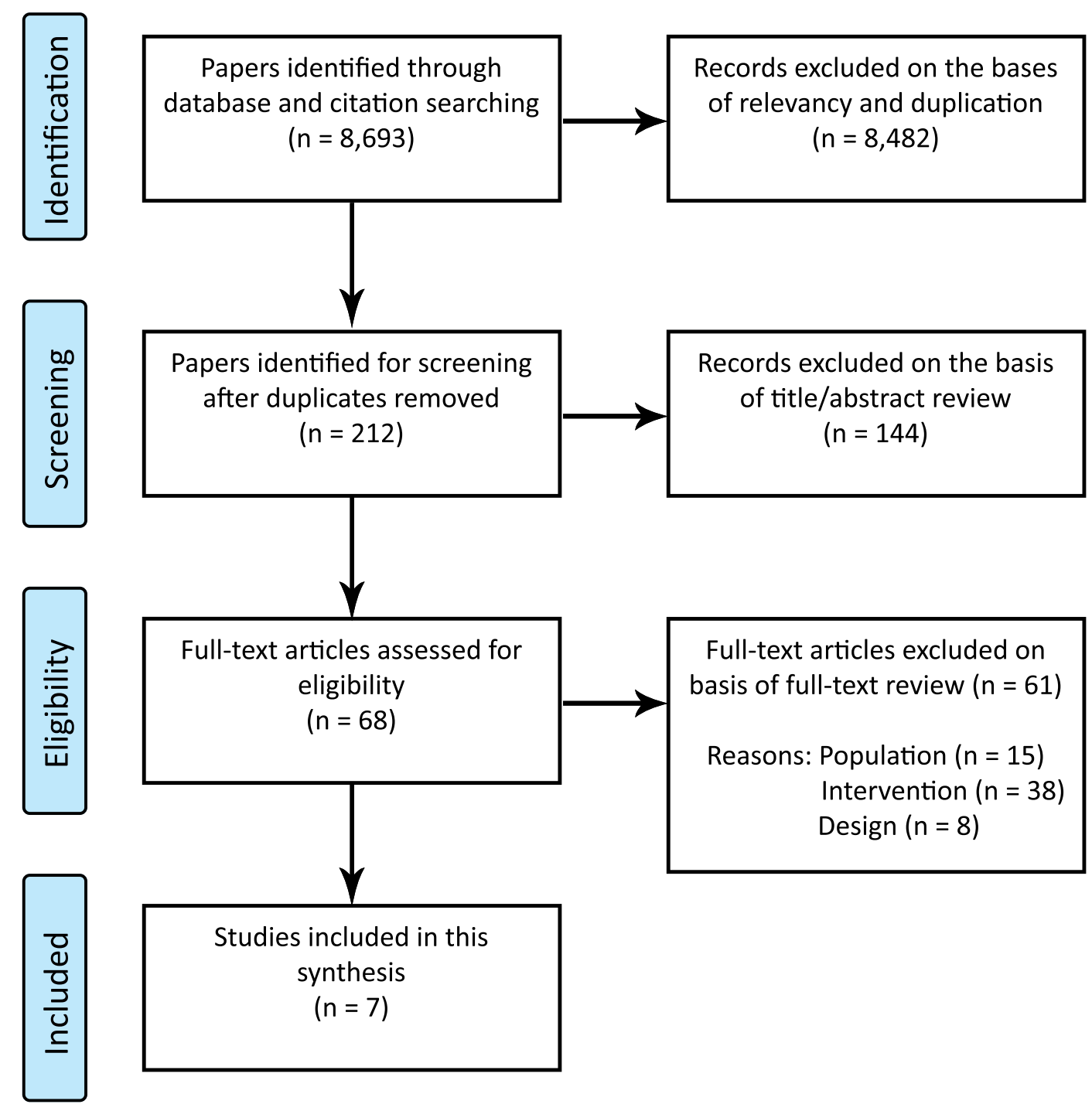

Figure 1. PRISMA (2009) flow diagram.

review was not intended to analyze specific treatment options nor look at out-patient services. Papers published in English language or having abstracts in English were included in this review.

With reference to the PICO framework (Population; Intervention; Comparison and Outcome), the Population included in this review were adult patients being admitted to or discharged from hospital. Interventions which focused on recovery and rehabilitation needs were included and encompassed hotels, and aftercare facilities. Comparison was made against traditional hospital care provision. Outcome measures were conceptualized from two perspectives; hospital expenditure in terms of length of stay, resource use, readmission rates; and patient experience in terms of satisfaction.

Whilst searching the literature, it became apparent that the term patient hotel/medical hotel was also used to describe facilities in the medical tourism sector. These establishments differ greatly, as both medical and surgical treatments are dispensed in this environment, as opposed to being used for non-acute care. For this reason, these studies were excluded from the review.

\section{Information sources and search strategy}

Studies were identified using bibliographic databases in relation to the research question. The research question for this review was: "What are the benefits a Patient Hotel model can bring to healthcare services in terms of cost and health outcomes to patients/guests as well as patient experience?" Initial searches were performed using Cochrane, Ovid Medline, CINAHL, Embase, ScienceDirect, Web of Science, and Scopus databases. Studies were included if they pertained to the patient hotel model, or similar model involving the integration of hospitality and healthcare design. The search included studies dating from $1^{\text {st }}$ January 2008 and was completed on 9th August 2018, to ensure included studies were current. The individual key search terms which were used in this review process were: "Recovery Hotel," "Patient Hotel," "Healthcare Hotel," "Meditel" and "Hospital Hotel." All quantitative and qualitative studies, including randomized controlled trials, quasi-experimental, pilot studies, cost analysis and feasibility studies were searched for to ensure all available evidence was captured.

\section{Search outcome}

The initial database search generated 8,679 papers, with an additional 14 papers retrieved from citation and hand searching, which culminated in 8,693 papers being retrieved. The search yielded a vast number of irrelevant articles owing to the 
commonality of the individual key search terms, despite a truncation technique being employed. From these 8,693 papers 8,481 articles were excluded due to duplication and relevancy, leaving 212 articles for screening. A full text eligibility screening process was carried out by two researchers independently, and a further 144 papers excluded. The final screening and eligibility process is depicted in Figure 1 using the PRISMA (2009) flow diagram [15].

\section{Data extraction and quality appraisal}

Titles and abstracts were screened for eligibility and all duplicates were removed. Papers were excluded if they lacked relevancy to patient hotels, or if they were not research studies. All decisions regarding eligibility, and selection of papers were resolved by consensus. The quality of the included studies was appraised using an accepted criterion for the evaluation of research [18]. Although debate surrounds the use of structured instruments for mixed methods reviews [19] structured instruments have shown reviewers make clear the explanations for their judgements [20]. Agreement in categorization of the papers was consistent across the reviewers.

\section{Data synthesis}

Due to the diversity in the descriptions, service provisions, and client groups the results were not subject to a meta-analysis. Data was synthesized, and themes and outcome measures were identified, these included: definition of a patient hotel, care provision, service user, patient experience, and cost analysis and effectiveness.

\section{Data collection and secondary analysis}

The results of the literature searches were scanned and reviewed by title, and abstract, with potentially applicable papers retrieved in full text format. Since this was a global synthesis of evidence, papers which had English abstracts available were translated into English language using a computerized translation application. Papers considered relevant to the search were reviewed by three academics and methodological rigor appraised using accepted criteria for the evaluation of research [18] and findings discussed collectively.

\section{Results}

Of 8,693 papers retrieved, 68 full-text articles were assessed for eligibility and from these seven articles were retained for screening. Out of these: One systematic review [6], and one Randomized Clinical Trial [5] were found. Other studies which have been included in this review offer a range of methodological approaches including one six-year retrospective analysis [21], one descriptive cost analysis study [22] one quantitative questionnaire [23] and two qualitative studies [12,24]. Table 1 shows the studies included in this review.

\section{Study characteristics}

The included studies were conducted in high income countries: Global studies $(n=1)$ England $(n=2)$ France $(n=1)$ Sweden $(n=2)$ Norway $(n=1)$. The total number of participants who directly participated in the selected studies was 232. Additionally, 11,871 patient stays were analyzed for suitability inclusion in patient hotel accommodation, to gain insights into cost

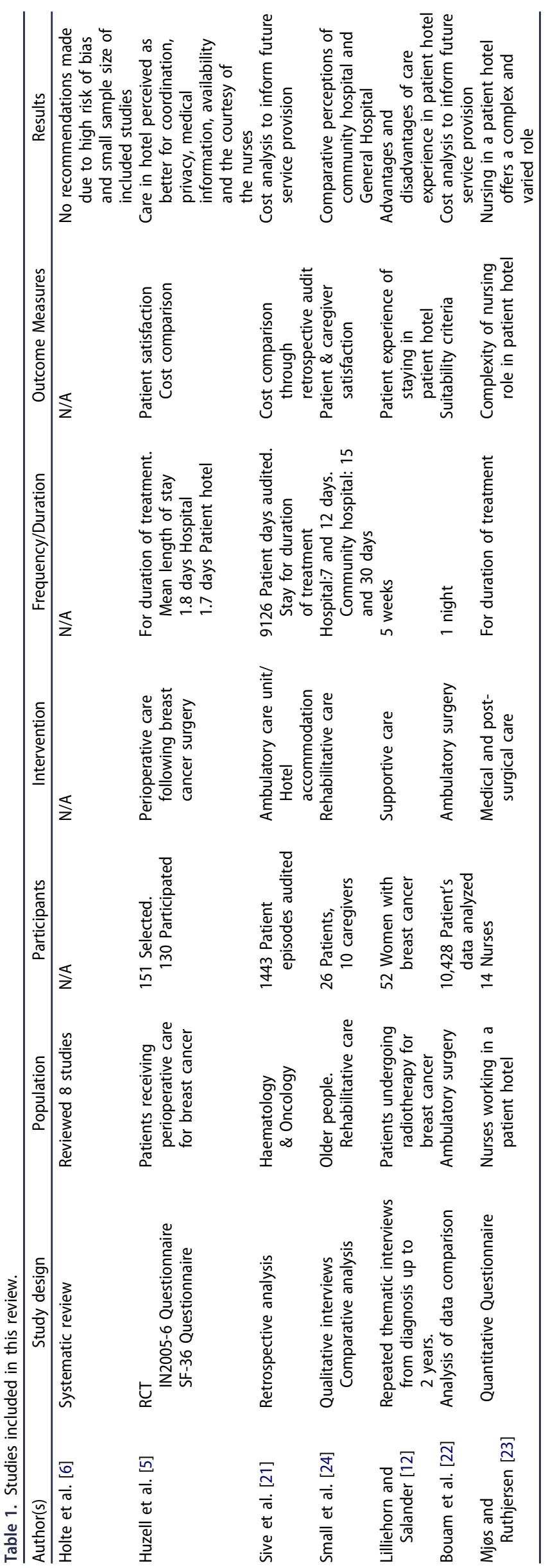


comparison and future service provision. The interventions of the selected studies were broadly heterogeneous in nature which made comparative analysis problematic. The service provisions included ambulatory surgery [22], ambulatory care for cancer patients [21] perioperative breast cancer care [5,12] postacute rehabilitation care for older people [24] medical and surgical care [23] and a systematic review of patient hotels [6]. The length of patient stay varied across the studies, depending upon the study aims and objectives, again making comparison problematic. The studies pertaining to cost analysis used a one-night stay to compare suitability and cost $[21,22]$. Where studies aimed to look at patient experience and patient satisfaction, this was mainly carried out based upon the entirety of their stay $[5,12,24]$. In a study looking at the nurse's role in patient hotels, a survey was carried out over a four-week period, involving fourteen nurses [23].

\section{Quality assessment of studies}

Quality appraisal of the included studies is described in Supplementary Table S1.

As noted above, a previously conducted systematic review by Holte et al. [6] concluded that due to the reviewed studies having small sample sizes and exhibiting a high risk of bias no recommendations could be drawn concerning the efficacy of the patient hotel compared to traditional hospital accommodation or in comparison to alternative treatment placements. Comparison across studies was problematic, as characteristics, methodology and results were not comparable. All the seven studies included in this review provided a description of the study sample size and sample characteristics and gave a justification of sample size and drop-out rates where applicable. A validated outcome measure was used in one of the studies [5]. Potential for bias was addressed in four studies $[5,12,21,24]$. Significance testing was used in one study [5]. However, the sample size in Huzell et al. [5] study was smaller than originally calculated through significance testing, and therefore the study could not attain the proposed value of patient experience score which was $15-20 \%$ higher compared to care in traditional hospital accommodation, therefore reducing the impact of the study [5]. Comprehensive cost analysis was not completed nor control group established in one study [21]. Likewise, in the study by Lilliehorn and Salander [12] there was also a lack of comparative data. In a quantitative study by Mjøs and Ruthjersen [23] a small sample size $(n=14)$ was utilized, making it difficult to assess the representativeness of the study.

\section{Outcome measures}

Outcome measures varied. Huzell et al. [5] utilized a validated questionnaire to evaluate perioperative care in two settings, while adjuvant findings in the study related the comparative costs of the two areas. Small et al. [24] utilized semi-structured interviews with staff to create vignettes describing care settings pertaining to community hospitals and general hospitals in England. The vignettes were then used in semi-structured interviews with patients and carers to explore perceptions of the respective accommodation and care [24]. In a qualitative study by Lilliehorn and Salander [12] the experiences of participants following perioperative breast cancer care were explored using repeated thematic interviews from diagnosis up to two years to examine the impact of living away from home in a patient hotel during periods of radiotherapy treatment. A study by Mjøs and Ruthjersen [24] used a pilot tested quantitative survey to evaluate the daily tasks involved in nursing at a patient hotel. In a descriptive study by Bouam et al. [22] the outcome measure concerned the comparative costs of using a patient hotel for ambulatory surgical patients and demonstrated that $52 \%$ of all patients matching the study criteria of suitability from the national database could be appropriate for this intervention. Sive et al. [21] completed a retrospective audit of ambulatory care unit (ACU) patients to assess the suitability and effectiveness of using a hotel-based ACU model, finding it to be a safe and efficient alternative to traditional hospitalization.

\section{Findings}

A systematic review by Holte et al. [6] was conducted for the National Knowledge Center for health services to inform future Norwegian service provision [6]. Holte et al. [6, p.6] concluded that no recommendations could be made due to the reviewed studies "including few patients and all of them having a high risk of bias". In a randomized clinical trial by Huzell et al. [5] no significant evidence was found in relation to the quality of care in a patient hotel compared to traditional hospital accommodation, although participants in the hotel group reported a better care experience with respect to privacy, courtesy and information giving. Patient satisfaction was also an outcome directly explored by Lilliehorn and Salander [12] which examined the impact of living in a patient hotel during periods of treatment. The study, by Huzell et al. [5] also reported cost efficiency in the accommodation at the patient hotel, which was found to be five times lower than that of traditional hospital accommodation. These findings were reflected in a study by Sive et al. [21] who found that a hotel-based ambulatory care unit model, provided both a safe, efficient and cheaper alternative to traditional hospitalization. Indeed, Bouam et al. [22] estimated cost savings to the French Healthcare system to be in the region of $€ 12.8$ million, by analyzing potential number of patients which were matched against suitability coding. In a qualitative study by Small et al. [24] a community hospital, which exhibited environmental benefits in keeping with the patient hotel model identified health outcomes linked to the greater independence which was available for participants. However, the study did not generate any differences in either patient satisfaction or cost comparison [24]. The nurses' role in a patient hotel, was explored by only one identified study [23] and provides limited evidence but suggests the role of the nurse in a patient hotel is varied and complex.

\section{Discussion}

Patient hotels are a relatively new concept which means that they have not become embedded in health service delivery on a global scale and this has meant that the quality of research is generally low. Studies have focused generally on the themes of patient experience and cost, to provide evidence to inform future service provision. Only one study [23] was found which explored the experience of nursing in a patient hotel, although the representativeness of this study is difficult to assess due to its small sample size. The overarching concern of the researchers conducting this review however lies in the fact that the patient hotel model has no clear definition, and inconsistencies in labeling are apparent. This may have impacted upon the review process whereby work may have been overlooked due to the diversity of classification.

The studies pertaining to patient experience reported that greater freedom, privacy and independence was found in the 
patient hotel model, in comparison to traditional hospital accommodation $[5,12,24]$. This may be because there is greater emphasis put upon self-care, and the involvement of family and friends, than in a traditional hospital environment, where patients are said to experience "pyjama paralysis" brought on by lengthy hospital stays and induced immobility [25, p.881]. The importance of the aesthetic environment was mentioned in a number of studies and served to reinforce the issue of consumer satisfaction and increased feelings of wellbeing $[5,24]$. The home-like qualities of the accommodation and involvement of relatives was found to be of particular value to older people where the focus was on rehabilitation [24]. In two studies, exploring the post-operative perceptions of women staying at a patient hotel following surgical intervention for breast cancer, both studies found that participants staying at a patient hotel experienced a greater sense of privacy and feelings of wellbeing, directly linked to the hotel-like environment $[5,12]$. However, the limited number of studies focusing on the subjective experiences of patients, family members and staff means that more research is needed to allow for greater insights into the benefits of the patient hotel model.

In evaluating the cost effectiveness of the patient hotel model, two studies focused directly on this measure of outcome [21,22]. Both studies used a retrospective audit approach, although Bouam et al. [22] conducted a matched comparison of the national hospital activity data to obtain results. The results from both studies demonstrated potential savings in using the patient hotel model for ambulatory surgical patients. In the audit by Sive et al. [21] the comparative cost of providing nursing care in a patient hotel is almost a third less, and savings were also highlighted in respect of increasing ambulatory care capacity, and therefore decreasing traditional hospital inpatient care by an additional 1844days, whilst freeing up hospital beds for patients requiring acute medical care. The study by Bouam et al. [22] found potential savings of over $€ 2.8$ million. A third study [5] found comparative savings in using a patient hotel against traditional hospital accommodation to be five times cheaper for perioperative care for patients following breast cancer surgery. The studies included in this review offer insights into the potential cost effectiveness of the patient hotel model, although further robust studies are needed to offer conclusive recommendations.

\section{Implications for service provision}

The combination of hospitality and healthcare is highly relevant to health service providers, who are now becoming attuned to patient satisfaction, as a quality of care indicator [26]. The literature reviewed reveals that the patient hotel model has the potential to offer health providers a cost-effective alternative for perioperative care, particularly in respect of ambulatory surgery $[21,22]$.

The improvement of patient flow though hospitals, to home, or non-acute care environments is a global problem for health service providers [27]. Delayed transfers of care have implications to health outcomes for patients awaiting transfer, as well as those requiring acute care and those on waiting lists [28]. An example of this is seen in the UK where there is a defined problem of delayed transfers of care, at the interface between acute and primary care, where patient flow is unnecessarily blocked as patients wait for non-acute NHS beds and social care to become available $[29,30]$. The use of patient hotels in this instance could provide a cost-effective alternative for these patients, who require either rehabilitation or recuperation, and not an acute medicalised environment.

\section{Limitations of this review}

This systematic review was unable to complete a meta-analysis of the quantitative data due to the diversity in descriptions, service provision and client groups relating to patient hotels. The many different labels applied to the patient hotel concept, include: meditel, recovery hotel, medical hotel, ambutel, hospital hotel and healthcare hotel. This diversity in description, function, and title means that comparative analysis is both problematic and complex. On reflection a mixed methods tool may have been more helpful in appraising the included studies. It is also acknowledged that papers published in languages other than English, with no English abstract, may not have been included in this review.

\section{Conclusions}

Due to the lack of studies available, this review can offer no recommendations pertaining to the efficacy of the patient hotel model, although there does appear to be a growing argument to suggest that patient hotels may offer positive benefits in terms of patient experience and cost effectiveness. More research in this area is needed to establish a body of evidence relating to the efficacy of health outcomes and cost benefits to healthcare providers, in comparison to traditional hospital accommodation. There is a need to focus on patient experience, staff experience, and the experiences of family/friends. Moreover, there needs to be more RCTs, with research that can be subject to international comparison. Research utilizing more methodologically diverse approaches would offer greater insights into the subjective experience of patients using the patient hotel model and could further assess the interventions provided.

\section{Disclosure statement}

The authors report no declarations of interest.

\section{ORCID}

Lorna Chesterton (D) http://orcid.org/0000-0002-9668-1941

\section{References}

[1] Bush H. Hospitals embrace hotel-like amenities: ensuring that patient-friendly services match clinical goals is a big challenge. H\&HN Hosp Health Netw. 2007;81:24.

[2] Steele JR, Jones AK, Clarke RK, et al. Health care delivery meets hospitality: a pilot study in radiology. J Am Coll Radiol. 2015;12:587-593.

[3] Zygourakis CC, Rolston JD, Treadway J, et al. What do hotels and hospitals have in common? How we can learn from the hotel industry to take better care of patients. Surg Neurol Int. 2014;5:S49-S53.

[4] Dijkstra K, Pieterse M, Pruyn A. Physical environmental stimuli that turn healthcare facilities into healing environments through psychologically mediated effects: systematic review. J Adv Nurs. 2006;56:166-181.

[5] Huzell MAC, Frisack J, Dalberg K. Randomized clinical trial comparing perioperative care for breast cancer patients at a patient hotel versus a general surgical ward. Patient Experience J. 2015;2:153-163.

[6] Holte $\mathrm{HH}$, Harboe I, Vist GE. Effect of patient hotels for hospitals and patients. Systematic review. Rapport fra 
Kunnskapssenteret nr 11-2011. Oslo: Nasjonalt kunnskapssenter for helsetjenesten; 2011 [cited 2019 Jun 6]. Available from: https://www.ncbi.nlm.nih.gov/pubmedhealth/PMH0097717/pdf/PubMedHealth_PMH0097717.pdf

[7] Wu Z, Robson S, Hollis B. The application of hospitality elements in hospitals. J Healthcare Manag. 2013;58:47-62.

[8] Pizam A. The "ity" factor. Int J Hospitality Manag. 2007; 26:499-501.

[9] Jha AK, Orav EJ, Zheng J, et al. Patients' perception of hospital care in the United States. N Engl J Med. 2008;359: 1921-1931.

[10] Hollis B, Verma R. The intersection of hospitality and healthcare: exploring common areas of service quality, human resources and marketing. Cornell Hospitality Roundtable Proceedings. 2015;4:6-15.

[11] Docrates. Healthcare hotel Helsinki. 2012 [cited 2018 Jun 22] Available from: http://www.docrates.com/en/docrateshospital/health-care-hotel-helsinki

[12] Lilliehorn S, Salander P. Living at a residency away from home during radiotherapy as narrated by 52 patients with breast cancer: a cage of safety and discomfort. Disabil Rehabil. 2018;40:450-456.

[13] Beckmann K, Abell L, Edwards D, et al. Providing accommodation services for rural cancer patients: the experience in South Australia. Cancer Forum. 2007;31:98-104.

[14] Nesje SB. Patient hotel without dressing gown and white coats. Tidsskr nor Laegeforen. 2000;10:1816.

[15] Liberati A, Altman DG, Tetzlaff J, et al. The PRISMA statement for reporting systematic reviews and meta-analyses of studies that evaluate healthcare interventions: explanation and elaboration. BMJ. 2009;339:b2700.

[16] NICE. Developing NICE guidelines: the manual; 2017 [cited 2019 Jun 6]. Available from: https://www.nice.org.uk/process/ pmg20/chapter/identifying-the-evidence-literature-searching-and-evidence-submission

[17] McElroy LM, Ladner DP. Defining the study cohort: inclusion and exclusion criteria. In: Pawlik T, Sosa J, editors. Success in academic surgery: clinical trials. Success in academic surgery. London: Springer; 2014. p. 131-139

[18] CASP. Critical Appraisal Skills Programme; 2018 [cited 2019 Jun 6]. Available from: https://casp-uk.net/casp-toolschecklists/

[19] Hannes K, Lockwood C, Pearson A. A comparative analysis of three online appraisal instruments' ability to assess validity in qualitative research. Qual Health Res. 2010;20: 1736-1743.

[20] Dixon-Woods M, Sutton A, Shaw R, et al. Appraising qualitative research for inclusion in systematic reviews: a quantitative and qualitative comparison of three methods. J Health Serv Res Policy. 2007;12:42-47.

[21] Sive J, Ardeshna KM, Cheesman S, et al. Hotel-based ambulatory care for complex cancer patients: a review of the University College London Hospital experience. Leuk Lymphoma. 2012;53:12,2397-2404.

[22] Bouam S, Gaucher S, Matrella F, et al. Increasing ambulatory surgery potential by non-medicalized accommodation: matched comparison of the 2011 national hospital activity data to 66 local stays. J Visc Surg. 2014;151:263-268.

[23] Mjøs EB, Ruthjersen AL. Hva er sykepleie på et internt pasienthotell? Nordisk sygeplejeforskning 2013;3:59-70.

[24] Small N, Green J, Spink J, et al. Post-acute rehabilitation care for older people in community hospitals and general hospitals - philosophies of care and patients' and caregivers' reported experiences: a qualitative study. Disabil Rehabil. 2009;31:1862-1872.

[25] Surkan MJ, Gibson W. Interventions to mobilize elderly patients and reduce length of hospital stay. Can J Cardiol. 2018;34:881-888.

[26] Anhang Price R, Elliott MN, Zaslavsky AM, et al. Examining the role of patient experience surveys in measuring health care quality. Med Care Res Rev. 2014;71:522-554.

[27] Shi P, Chou MC, Dai JG, et al. Models and insights for hospital inpatient operations: time-dependent ED boarding time. Manag Sci. 2015;62:1-28.

[28] Kingsfund. Delayed transfers of care: a quick guide; 2018 [cited 2019 Jun 6]. Available from: https://www.kingsfund. org.uk/publications/delayed-transfers-care-quick-guide

[29] NHS England. The forward view into action: planning for 2015/16; 2014. [cited 2019 Jun 6]. Available from: www. england.nhs.uk/wp-content/uploads/2014/12/forward-viewplning.pdf

[30] NHS England, Care Quality Commission, Health Education England, Monitor, Public Health England, Trust Development Authority. NHS five year forward view. London: NHS England. 2014 [cited 2019 Jun 6]. Available from: www.england.nhs.uk/ ourwork/futurenhs/ 